\title{
Neisseria flavescens
}

National Cancer Institute

\section{Source}

National Cancer Institute. Neisseria flavescens. NCI Thesaurus. Code C86602.

A species of aerobic, Gram-negative, diplococci shaped bacteria assigned to the phylum Proteobacteria. This species is catalase and oxidase positive, non-hemolytic, pigmented, susceptible to colistin, produces polysaccharides from sucrose, and does not produce acid from glucose, maltose, fructose, sucrose, mannose or lactose. N. flavescens is commensal in the human oral cavity and can become pathogenic in immunocompromised individuals causing endocarditis, mening itis, and septicemia. 